\title{
Broadening and strengthening stakeholder engagement in emergency preparedness, response and recovery
}

\author{
R. Geysmans ${ }^{1, *}$, N. Zeleznik ${ }^{2}$, B. Abelshausen ${ }^{1}$, T. Duranova ${ }^{4}$, C. Schieber ${ }^{3}$, T. Schneider ${ }^{3}$, P. Crouail $^{3}$, \\ C. Turcanu ${ }^{1}$, L. Liutsko ${ }^{5}$ and M.C. Cantone ${ }^{6}$ \\ 1 SCK CEN-Belgian Nuclear Research Centre, Boeretang 200, B-2400 Mol, Belgium. \\ 2 EIMV-Milan Vidmar Electric Power Research Institute, Hajdrihova 2, 1000 Ljubljana, Slovenia. \\ ${ }^{3}$ CEPN-Centre d'étude sur l'Évaluation de la Protection dans le domaine Nucléaire, Fontenay-aux-Roses, France. \\ ${ }^{4}$ VUJE, Okruzna 5, 91864 Trnava, Slovak Republic. \\ ${ }^{5}$ ISGlobal, Dr. Aiguader, 88, 08003 Barcelona, Spain. \\ ${ }^{6}$ UMIL-University of Milan, Via Pascal 36, 20133 Milan, Italy.
}

\begin{abstract}
This article discusses stakeholder engagement in the field of nuclear and radiological emergency preparedness, response, and recovery (EPR \& R). It argues that stakeholder engagement in this field could be broadened - in terms of stakeholders and forms of engagement, and strengthened - in terms of sustainability and impact. Four different dimensions are identified and presented along which this process of broadening and strengthening can occur: (1) the underlying rationales of engagement, (2) top-down and bottom-up engagement, (3) formal and informal engagement, and (4) radiological protection culture. Based on the analysis of these dimensions, a reflection on power and non-participation is provided, and recommendations on stakeholder engagement are formulated.
\end{abstract}

Keywords: nuclear and radiological emergencies and post-accident exposures / stakeholder engagement / participation / ENGAGE

\section{Introduction}

Preparedness for, response to, and recovery from, a nuclear or radiological emergency is characterised by a complex and tangled web of provisions and actions, in which many stakeholders may have a role or interest (Heriard-Dubreuil et al., 2010; Heriard-Dubreuil and Baudé, 2016). These roles or interests of stakeholders are moreover highly diverse, and can encompass for instance: having a legal or professional role in emergency or recovery management (e.g. first responders, national and local authorities); being directly or indirectly impacted by the accident (e.g. communities and local population working or living near a nuclear power plant); taking up a role as a proxy for other stakeholders (e.g. NGOs representing the environment).

The complexity of nuclear emergency preparedness, response and recovery (EPR \& R) partly originates from the unpredictability of events and their consequences. In this sense, it is a challenging task to identify specific stakeholders, given the speculative state of events. Complexity also stems

\footnotetext{
*Corresponding author: robbe.geysmans@sckcen. be
}

from the fact that both the preparedness for, and the management of, an actual emergency comprises a range of decision-levels (e.g. local, regional, (inter)national), time frames (e.g. short-term emergency responses and longer-term recovery actions), risks and interests (e.g. for local populations, institutional actors, consumers, animals, plants, etc.) (French et al., 2007; Marignac et al., 2016). In short: the (potential) impacts of a nuclear emergency "are felt across society in a plethora of ways and require many perspectives if we are to handle them in sensitive, acceptable ways" (French et al., 2007, p. 422).

Although identifying stakeholders in EPR \& $\mathrm{R}$ is complex and challenging, previous nuclear emergencies increased awareness on the importance of risk communication and stakeholder engagement in emergency management, as a lack of communication and engagement can result in inadequate responses and delayed recovery (Lochard, 2013; Perko, 2016; Sato, 2016; Schneider et al., 2019). This awareness is reflected in a number of guidelines that call for stakeholder engagement in nuclear EPR \& R (e.g. ICRP, 2009a, 2009b; WHO, 2017).

Some elements found in these international guidelines are also actualised in (inter)national regulatory frameworks and legal prescriptions (Zeleznik et al., 2019a). The 2013 EU Basic 
Safety Standards directive for example requires consultation with stakeholders and their involvement in decision-making in existing exposure situations, including post-accidental situations (2013/59/EURATOM). However, the understandings of who the stakeholders are, and the descriptions of how they can be engaged in EPR \& $R$ are often quite narrow or left unelaborated in existing legal frameworks. This relates to a more general observation from the field of science and technology studies that dominant understandings and conceptions of stakeholder participation have often been rather limited in terms of their framing or scope (Wynne, 2007; Wehling, 2012).

In an effort to identify opportunities for broadening and strengthening stakeholder engagement, the ENGAGE project has studied existing guidelines for and practices of stakeholder participation in the field of EPR \& $R^{1}$. Broadening in this context refers to a widening of current dominant understandings and conceptions of stakeholders and the ways in which they could engage. Strengthening denotes the ways in which stakeholder engagement can become more sustainable (in terms of its robustness and durability) and impactful (in terms of its effects on emergency preparedness, response and recovery). In this paper, four dimensions are introduced along which such broadening and strengthening can be realized. These dimensions focus on (1) the rationales for engagement, (2) formal and informal stakeholder engagement, (3) top-down and bottom-up engagement, and (4) the development Radiological Protection (RP) culture.

After a short presentation of the methods used in the ENGAGE project, each dimension is elaborated upon in more detail. In the discussion section, attention is directed to the power dynamics which shape stakeholder engagement, and the issue of non-participation. The article concludes with a set of recommendations for stakeholder engagement in the field of EPR \& R.

\section{Methods: analyzing prescriptions, expectations and practices of stakeholder engagement}

To get insight in the existing legal prescriptions, guidelines and expectations regarding stakeholder engagement in nuclear emergency management, a variety of recommendations and (inter)national legislative documents were analysed, particularly focusing on whether and how stakeholders and stakeholder engagement are defined and prescribed. Different European Council Directives were studied, ranging from the 2013 Basic Safety Standards (2013/59/EURATOM) to more specific directives (2013/51/EURATOM; 2009/71/EURATOM; 89/618/EURATOM). National legislative documents relevant to nuclear or radiological EPR \& $\mathrm{R}$ were also analysed in four European Member States (Belgium, Italy, Slovenia, and Spain). Furthermore, a range of reports and guidelines from different international organizations were identified and studied. These comprise documents from the IAEA, HERCA, OECD-NEA, ICRP, WHO, NERIS and Nuclear Transparency Watch (NTW) (Zeleznik et al., 2019a). Finally, three inter-

\footnotetext{
${ }^{1}$ Also the field of indoor radon and medical exposure were studied in the ENGAGE project.
}

views focused on international experts' expectations regarding stakeholder engagement in EPR \& R.

Furthermore, attention was directed to actual practices of stakeholder engagement in the field of (preparedness for) emergency management. This was done through a variety of case studies, in different European countries. These aimed to gain insight into whether and how the identified guidelines, legal prescriptions and expectations were translated into practice, how radiation protection communities react to them, and which different stakeholders and forms of engagement can be found in practice, also beyond existing guidelines and legal prescriptions. Common analytical focal points included: stakeholder definitions, rationales for engagement, levels of involvement (e.g. one-way communication, gathering information, dialogue, collaboration, empowerment), initiators and outcomes of the process. For an overview of case studies, see Table 1 (see Zeleznik et al. (2019b) and Turcanu et al. (2020) for additional details).

In addition, another set of case studies was conducted, focusing on radiological protection (RP) culture (see Turcanu et al., 2020 for an elaborate definition). These case studies investigated how RP culture is characterised, which processes can build and enhance it, and how stakeholders can be engaged in/through it. An interest was taken in how developing RP culture might connect to more robust stakeholder engagement and informed decision-making in EPR \& R. For an overview of different case studies on RP culture, see Table 2 (see Barazza et al. (2019) for additional details).

Two workshops were organised to interact with stakeholders, and to collect their comments and questions related to the work done in the ENGAGE project. The first workshop was conducted in Athens (February 13-15, 2019) and the second in Bratislava (September 11-13, 2019).

\section{Broadening and strengthening stakeholder engagement}

Scholars working on public participation in science, innovation and technology have argued that dominant understandings and conceptions of stakeholder participation are often limited in their framing of who participates, how, and why (see e.g. Wynne, 2007; Felt and Fochler, 2010; Wehling, 2012). Our analysis of guidelines and practices concerning the engagement of stakeholders in EPR \& $R$ also recognized certain limited dominant understandings and conceptions. We then identified opportunities for broadening these, as well as ways in which stakeholder engagement can be strengthened. In the following sections, four different dimensions are presented along which this dynamic of broadening/strengthening can be realized, focusing respectively on the rationales for engagement, formal and informal stakeholder engagement, top-down and bottom-up engagement, and RP culture.

\subsection{Recognizing the different rationales of engagement}

Stakeholder engagement can be motivated by a range of imperatives or rationales. Three main rationales have been recognized and described in the literature; an instrumental, a substantive and a normative rationale (Fiorino, 1990; Stirling, 2005). 
Table 1. Overview of case studies on stakeholder engagement practices.

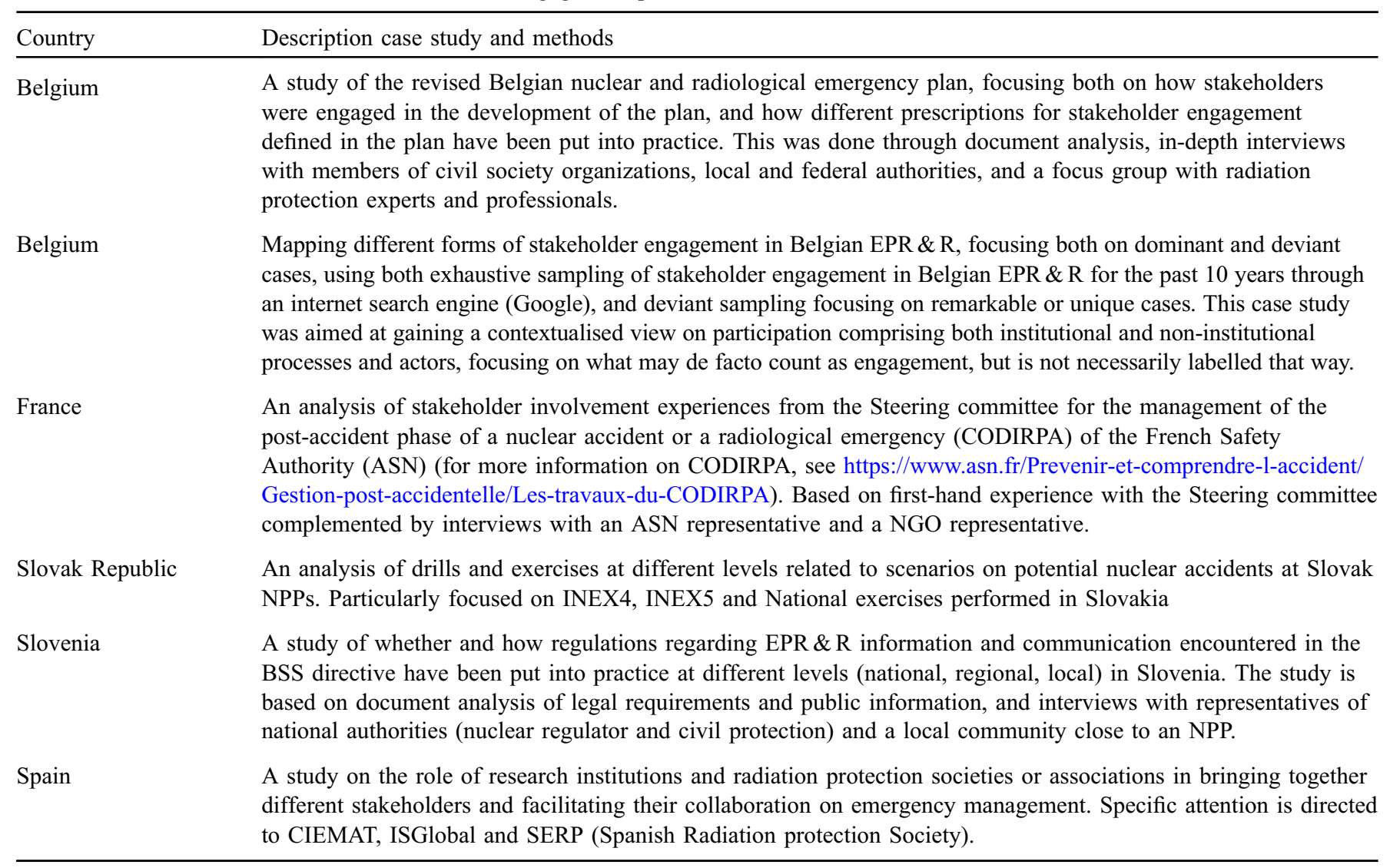

Table 2. Overview of case studies on RP culture.

\begin{tabular}{|c|c|}
\hline Country & Description of case study \\
\hline Slovak Republic & $\begin{array}{l}\text { Study of building of RP culture through actions (workshops, seminars, training courses, technical visits) undertaken } \\
\text { to improve and strengthen the emergency and post-accident preparedness and recovery management at national, } \\
\text { regional and local levels. These actions were part of European and national projects enhancing EPR \& R. }\end{array}$ \\
\hline
\end{tabular}

Each of these rationales should be understood as a specific "system of justification for participation", which connects to "choices for specific structures and procedures" (Wesselink et al., 2011,p. 2689). The normative rationale builds on the idea that stakeholder engagement is the "right thing to do", and as such relates to ethical and democratic values of justice and fairness (Stirling, 2005). In an EPR \& R context, an example of a normative rationale can be found in ideas that citizens living close to a nuclear facility have a right to be involved in decision-making on emergency preparedness, as they could be directly affected. The substantive rationale builds on the idea that different stakeholders have different experiences, expertise, or frames of reference, and that bringing these together will lead to improved decisions, policies, or assessments. In emergency and recovery management, this could for example mean that local first 
responders, authorities, and key representatives of civil society are actively involved in planning emergency responses during the preparedness phase, as these actors have first-hand knowledge and experience with the specificities of a particular local context. And thirdly, the instrumental rationale connects to the idea that stakeholder engagement is a valid or good way to reach specific goals, thereby envisaging engagement as a "means to an end". Engaging citizens in campaigns on what to do in an emergency situation, so they are more willing to accept certain measures in case of an actual emergency, is an example of how an instrumental rationale shapes stakeholder engagement in EPR \& R.

When analysing the different (inter)national guidelines, recommendations and regulations on emergency management, and the prescriptions these entail regarding stakeholder engagement, it is not always easy or possible to discern the particular rationales underlying or justifying stakeholder engagement. However, focusing on the actions which are prescribed in many of these documents, and the ways these are occasionally explicitly justified, the instrumental rationale seems to be dominant (Zeleznik et al., 2019a). To illustrate this claim, two examples can be provided. First, in the $2013 \mathrm{EU}$ BSS Directive, emphasis is put (inter alia) on information provision to members of the public likely to be affected in case of a nuclear emergency, so they are aware of "the action they should take in the event of such an emergency" (2013/59/ EURATOM, p. 30). And as a second example, the Belgian nuclear and radiological emergency plan mentions how in the recovery phase, stakeholders should be engaged through consultation, aimed at attaining as fast as possible a return to "normal" living conditions for the population of the affected area.

In both examples, the main emphasis is on the effectiveness of actions, and stakeholder engagement is presented as a means to attain this goal. And although some hints at normative and substantive rationales can arguably be detected (e.g. citizens should be informed in the preparedness phase because they have a right to know, or different stakeholders should be involved because this leads to an improved recovery after the emergency), these rationales are less explicitly present. This is noteworthy because each rationale holds a particular view of what stakeholder engagement could deliver, and thus connects more closely to particular forms and procedures of engagement (Wesselink et al., 2011).

We argue that mobilising a combination of the different rationales in both prescriptions and practices opens possibilities for engaging a wider range of stakeholders, including also those that may otherwise be left out, in a larger array of engagement forms and levels, better reflecting the needs of the different stakeholders (Turcanu et al., 2019). Recognising the ethical values underlying engagement, and its contribution to the quality of decision-making, might also make engagement more sustainable and impactful, as it gives a more equal weight to process and outcome. Overall, combining rationales would entail that stakeholder engagement is organised along the different rationales, that debate is stimulated on the motivations for engagement in the process of elaborating and implementing emergency plans, that reference is made to the different underlying rationales in prescriptions and guidelines, and that stakeholder engagement is connected to a critical reflection on underlying motivations at regular intervals to keep the debate going, reveal and challenge assumptions, and create new insights (Turcanu et al., 2019).

\subsection{Top-down and bottom-up initiation of participation}

A second dimension along which a broadening and strengthening of stakeholder engagement can be achieved, is through recognizing and embracing both top-down and bottom-up forms of engagement in preparedness for and recovery from a nuclear emergency.

The distinction between bottom-up and top-down in this context refers to the initiator(s) of the process: who is/are able to set the (initial) goals, motivations, and procedures which frame stakeholder engagement? Whereas top-down engagement is initiated by an institutional authority at the "top" (e.g. (inter)national governmental agencies), bottom-up engagement is initiated by local actors (e.g. citizens) who act according to their own perceived needs (Powell and Colin, 2009).

Interestingly, the forms of stakeholder engagement analysed in the case studies are mostly initiated top-down. Examples include emergency exercises, question \& answer sessions, or prescriptions for and efforts regarding communication and information at local, regional and national levels.

If top-down stakeholder engagement is organized in an open and flexible way, it holds potential for engaging a range of stakeholders (cfr. Powell and Colin, 2009). CODIRPA in France (the steering committee for the management of the post-accident phase of a nuclear accident) for example demonstrates how top-down initiatives do not necessarily preclude the involvement of stakeholders in different steps of the process, including setting its form and goals. Case studies in France and Slovakia have shown how top-down engagement, when accompanied by a strong commitment of the regulatory authority, can be both impactful and sustainable. Other case studies, however, have highlighted nevertheless two opportunities for broadening and strengthening stakeholder engagement in emergency and recovery management.

First, in the preparedness phase, many top-down initiatives seem to adhere to a distinction between those stakeholders who are "professionally" involved due to the role they are assigned because of the expert function they execute, and those stakeholders who are not. The latter encompasses different publics (e.g. local inhabitants, consumers, producers, etc.) or representatives of these publics (e.g. NGO's). The forms of engagement foreseen for these different stakeholder categories is then differentiated according to their perceived levels of expertise, and predefined by the actors who initiate engagement. Such distinctions, and the assumptions which are at their basis, should be a topic of reflection, in order not to exclude certain stakeholders or certain forms of engagement a priori, especially as this risks a mismatch between levels of engagement and stakeholders' needs and wishes for involvement, and thus could threaten sustainable, constructive and impactful engagement. This reflection may reveal new stakeholders, such as parents with children in schools or health professionals in hospitals, which, through engagement in the preparedness phase can provide a reality check to emergency and recovery plans. 
Second, while the dominant focus is on top-down engagement, forms of bottom-up initiation can also be found, and this both in preparedness for and in recovery from nuclear emergencies. For example, the Belgian local partnerships on radioactive waste management have initiated local campaigns aimed at informing their communities of the actions to be taken in case of a nuclear emergency. And in Japan, after the Fukushima-Daiichi disaster, local citizens and volunteers were quite prompt in setting up measurement and monitoring initiatives (cfr. blog.safecast.org; Kenens, 2020). However, regarding this latter example, although the potential contribution of citizens' measurements to emergency response has been acknowledged (Baudé et al., 2016), it is not yet reflected in national policies. We can thus argue that recognizing bottom-up engagement in both prescriptions and practice, identifying it as a valuable complement to top-down forms, and taking its outcomes into account in EPR \& R policies and strategies are important steps towards broadening the dominant conceptions of stakeholder engagement.

\subsection{Formal and informal engagement}

Closely related to the distinction between top-down and bottom-up engagement (focused on who initiated the activity), is the distinction between formal and informal engagement. "Formal" stakeholder engagement is organized along the lines of formal requirements and prescriptions, which define the frames within which engagement is to take place. "Informal" stakeholder engagement, in contrast, comes into existence independent of formal prescriptions or requirements (Hassenforder et al., 2018). It entails self-organizing initiatives, along the frames set by the involved stakeholders. In this sense, the distinction formal/informal partially runs along the lines of "invited" versus "uninvited" public participation described by Brian Wynne (2007), as uninvited public participation refers to the ways in which "citizens independently mobilize themselves, according to their own concerns, meanings, resources and issues" (Wynne, 2007, p. 104).

Two ways can be identified in which broadening and strengthening stakeholder engagement can happen with respect to formal/informal engagement. First, stakeholder engagement in emergency management is primarily focused on formal engagement, especially in the preparedness phase. However, case studies suggest that not all stakeholders wish to be formally engaged. Furthermore, case studies also revealed forms of informal stakeholder engagement that can/do play an important role in both emergency preparedness and recovery. Examples include local citizens which have organized themselves around a "nuclear artwork" in Belgium, citizens' radiation monitoring networks around NPPs (e.g. TDRM, tdrm.fiff.de/index.php/nl/), citizen science in post-Fukushima Japan or representatives of civil society in the Nuclear Transparency network who made independent assessments of EPR \& R in Europe. Informal exchanges, ad hoc groups and contacts with and between authorities and experts can also be placed under this header of "informal" engagement. Moreover, different informal engagement initiatives have explicitly pleaded for a better recognition of their existence and the output they generated (e.g. data), and thus want their voices to be heard (Zeleznik et al., 2019b). Better recognition would then entail the identification of informal stakeholder engagement by emergency management organisations, the provision of space for these informal initiatives and networks to develop, and the establishment of opportunities and pathways for collaboration and mutual reinforcement of formal and informal engagement.

Second, provided that formal engagement is organised along the lines of formal requirements and prescriptions, a way to strengthen and broaden engagement is the more detailed elaboration of stakeholder engagement in these formal requirements. Integrating stakeholder engagement into emergency and recovery policies and plans through a more elaborate description of forms and processes of engagement, and a clearer attribution of responsibilities for initiating, funding and maintaining these initiatives would be an important step in this direction. For example, case studies in Spain have demonstrated a lack of continuity in the preparedness phase if actions are too rigidly dependent on specific individual persons (who might retire, change jobs, etc.) or organizations which do not have continuous funding. Currently, nuclear emergency plans do not specify how stakeholder engagement should be organised at (inter)national, regional, and/or local levels. This implies a lack of clarity on who should take on the initiative to set up stakeholder networks in the preparedness phase, or how to engage with stakeholders in post-emergency management (Zeleznik et al., 2019b). A special attention point should be the openness (stakeholders can opt in and out of engagement) and flexibility (stakeholders define the goals, outcomes and process) of these engagement processes.

\subsection{Stakeholder engagement in/through RP culture}

A final identified dimension along which broadening and strengthening of stakeholder engagement can take place, relates to the development of radiological protection (RP) culture. RP culture refers to a set of perceptions, values, attitudes, beliefs and expectations related to radiation risk; an assembly of knowledge, know-how, skills, experience, and practices related to radiological protection; and a dynamic building process based on multi-stakeholders' interactions (Barazza et al., 2019; Turcanu et al., 2020 for an elaborate definition). The development of RP culture is particularly relevant during the preparedness phase of the emergency/postaccident situation. There is a large variety of stakeholders, at the local, national or international level, who should be involved in emergency preparedness: public authorities, elected representatives, civil protection, firefighters, health professionals, parents, teachers, economic actors, representatives of the population, NGOs, students and others. Many of them have no direct experience in the field of nuclear emergency management. It is then crucial to develop RP culture among these stakeholders to enable them to cope with the complexity of post-accident management and to build capacities to participate and to interact in the process of elaboration of EPR \& R plans and exercises.

$\mathrm{RP}$ culture is inherently connected to stakeholder engagement, as it (1) relates to and encompasses stakeholders' perceptions, values, actions, know-how, and behaviours regarding radiation protection, and (2) is developed through 
the engagement of stakeholders. Case studies highlighted various processes contributing to the development of RP culture in EPR \& R (Barazza et al., 2019). In most cases, these are integrated into participatory processes set up for the preparation of emergency response and recovery. Specific forms include, among other, working groups, seminars, training sessions, or nuclear emergency exercises.

The CODIRPA process in France (https://www.asn.fr/ Prevenir-et-comprendre-l-accident/Gestion-post-accidentelle/ Les-travaux-du-CODIRPA) for example shows that developing RP culture in the preparedness for post-accidental situations relies largely on the capability to be connected to concrete cases and lessons learned from past experiences, which enables a better comprehension of the complexity of post-accident situations. This complexity entails that a broad range of stakeholders need to be involved, ranging from experts to national and local authorities, health professionals, local population, local elected people, and specific professionals. These stakeholders will play a key role in the emergency and recovery phases, and as such the establishment of radiological protection culture can lead to improved management of the situation.

\section{Discussion}

Starting from the idea that stakeholder engagement in nuclear emergency management can be broadened and strengthened by moving beyond dominant understandings and conceptions of stakeholders and engagement (which emphasise top-down, formal and instrumentally motivated engagement), this paper has discussed four dimensions along which broadening and strengthening can be achieved. Although contexts undeniably vary, we believe that the suggestions made are applicable to a wide variety of situations related to nuclear EPR \& R. In this section, there are two issues which will be further discussed: power and non-participation.

Highlighting how some understandings and conceptions of stakeholders and engagement seem to be dominant in the current field of nuclear emergency management, implicitly refers to the issue of power. Who participates, which processes are used, which needs, goals and rationales are perceived as underlying engagement, are all the object of framing and power dynamics (cfr. Stirling, 2005). Recognizing the ways in which power works on/through stakeholder engagement, highlights how engagement is not a "neutral" issue, and how certain forms are more in line with dominant power structures, while others challenge these (Young, 2001).

Regarding the different rationales for engagement discussed above, Stirling (2005) argues that each rationale takes a distinct position towards the issue of power. While the normative rationale seems fit to counter "undue exercise of power", instrumental rationales inscribe themselves in existing power structures, and substantive rationales are "ostensibly blind to considerations of power" (Stirling, 2005, p. 223). In Section 3 we argued for broadening and strengthening engagement through recognizing and including all three rationales for engagement in prescriptions and practice. This entails therefore a recommendation to reflect upon and recognize the different power dynamics underlying these rationales, and the ways in which using them can also confirm/ challenge existing power structures.
In a similar vein, formal and informal stakeholder engagement can each be connected to distinct power dynamics; whereas the former can be put in line with the expectations of powerful agents, the latter is more likely to be the resort of those lacking power in current society (Wynne, 2007; Wehling, 2012). Since formal engagement happens along lines set out by legal requirements and prescriptions, those who define these requirements also define the forms of engagement, both directly (through the coercive power of prescriptions), and indirectly (through using certain wordings and definitions, which shape dominant perspectives on stakeholder engagement).

This discussion of power also offers food for thought regarding the issue of non-participation, which is regularly encountered in nuclear emergency preparedness. Why do some stakeholders not participate in emergency management? A spectrum of possible explanations exists, many of which can be related to the aforementioned power dynamics. One explanation could be that some stakeholders are not perceived as stakeholders in the first place, and are thus not able to participate. This could be the consequence of unawareness, but also of an explicit strategy not to involve them. In the first case, attaining to a broad understanding of what a stakeholder could be and using different stakeholder identification strategies, offers a possible way out of such impediments to participation. In the second, informal and bottom-up engagement might offer possibilities for neglected stakeholders to raise their voices. Another reason why stakeholders might not participate, is because they lack the resources to do so, which again can be both a willing or unwilling outcome of power dynamics. Finally, stakeholders might not participate because they are not eager to do so, e.g. because of disinterest in the issue at hand, other priorities, or to express disagreement with the process or its framing. In the latter instance, this unwillingness might thus stem from a refutation to be inscribed in dynamics of power and dominance with which one does not agree (see e.g. Young, 2001). Whatever the reason for non-participation is, policymakers, researchers and practitioners need to reflect upon its causes. The right not to participate should be acknowledged, but the intended exclusion of stakeholders in processes which (could) affect them is of course not desirable. To the extent possible, different ways for moving "in" and "out" of participation, and thus having one's voice heard, should be enabled.

\section{Conclusions}

Reflecting the research conducted within the ENGAGE project, this article proposes ways in which stakeholder engagement in nuclear emergency management can be broadened and strengthened. More specifically, through focusing on four dimensions along which this broadening and strengthening could take place, it argues for a reflection on current guidelines for and practices of stakeholder engagement, and the challenges and opportunities they entail. Overall, this article supports the ENGAGE recommendations, which argue to:

1 broaden the motivation for stakeholder engagement in emergency preparedness, response and recovery, in both prescriptions and practice;

2 broaden the scope of "participation" in emergency preparedness, response and recovery; 
3 recognise the role of informal stakeholder engagement in EPR \& R;

4 integrate stakeholder engagement in EPR \& R plans and policies;

5 establish strategies for continuous, 2-way communication about emergency planning, tailored to specific stakeholders from both local and wider areas;

6 elaborate a strategy to foster the development of radiological protection culture in the preparedness phase.

Acknowledgements. The work described in this paper was conducted within the ENGAGE project, which was part of the H2020 CONCERT project. This project received funding from the EURATOM research and training programme 2014-2018 under grant agreement No. 662287.

Disclaimer (Art. $29.5 \mathrm{GA}$ ). This publication reflects only the author's view. Responsibility for the information and views expressed therein lies entirely with the authors. The European Commission is not responsible for any use that may be made of the information it contains.

\section{References}

Barazza F et al. 2019. Final report on case studies, including recommendations and guidelines on building and enhancing radiation protection culture. CONCERT Deliverable 9.87.

Baudé $\mathrm{S}$ et al. 2016. Local populations facing long-term consequences of nuclear accidents: Lessons learnt from Chernobyl and Fukushima. Radioprotection 51(HS2): 155-158.

Council Directive 89/618/EURATOM. 1989. Informing the general public about health protection measures to be applied and steps to be taken in the event of a radiological emergency.

Council Directive 2009/71/EURATOM. 2009. Establishing a Community framework for the nuclear safety of nuclear installations.

Council Directive 2013/51/EURATOM. 2013. Laying down requirements for the protection of the health of the general public with regard to radioactive substances in water intended for human consumption.

Council Directive 2013/59/EURATOM. 2013. Laying down basic safety standards for protection against the dangers arising from exposure to ionising radiation.

Felt U, Fochler M. 2010. Machineries for making publics: Inscribing and describing publics in public engagement. Minerva 48(3): 219-238.

Fiorino DJ. 1990. Citizen participation and environmental risk: A survey of institutional mechanisms. Sci. Technol. Human Values 15(2): 226-243.

French S, Carter E, Niculae C. 2007. Decision support in nuclear and radiological emergency situations: Are we too focused on models and technology? Int. J. Em. Man. 4(3): 421-441.

Hassenforder E et al. 2018. What's the middle ground? Institutionalized $v s$. emerging water-related stakeholder engagement processes. Int. J. Water Res. Dev. 35(3): 525-542.

Heriard-Dubreuil G, Baudé S. 2016. Supporting people building their own response to the consequences of a nuclear accident: Complexity management, trust and the Aarhus Convention. Radioprotection 51(HS2): S153-S154.

Heriard-Dubreuil G et al. 2010. The EURANOS cooperative framework for preparedness and management strategies of the long-term consequences of a radiological event. Radioprotection 45: S199-S213.

ICRP Publication 109. 2009a. Application of the Commission's recommendations for the protection of people in emergency exposure situations. Ann. ICRP 39(1).

ICRP Publication 111. 2009b. Application of the Commission's recommendations to the protection of people living in long-term contaminated areas after a nuclear accident or a radiation emergency. Ann. ICRP 39(3).

Kenens J. 2020. Changing perspectives: tracing the evolution of citizen radiation measuring organizations after Fukushima. Radioprotection 55(HS2). https://doi.org/10.1051/radiopro/2020012.

Lochard J. 2013. Stakeholder engagement in regaining decent living conditions after Chernobyl. Radioact. Environ. 19: S311-S331.

Marignac Y, Hazemann J, Baudé S. 2016. Managing the complexity of societal needs in a nuclear emergency situation: towards further experts collaboration for the "enlightened protection" of populations. Radioprotection 51(HS2): S159-S161.

Perko T. 2016. Risk communication in the case of the Fukushima accident: Impact of communication and lessons to be learned. Int. Env. Ass. Man. 12(4): 683-686.

Powell MC, Colin M. 2009. Participatory paradoxes: Facilitating citizen engagement in science and technology from the top-down? Bull. Sci. Tech. Soc. 29(4): 325-342.

Sato A. 2016. Nuclear disasters and risk communication: Learning from Fukushima. Policy Brief 5.

Schneider T et al. 2019. The role of radiological protection experts in stakeholder involvement in the recovery phase of post-nuclear accident situations: Some lessons from the Fukushima-Daïchi NPP accident. Radioprotection 54(4): 259-270.

Stirling A. 2005. Opening up or closing down: Analysis, participation and power in the social appraisal of technology. In: Science and citizens: Globalization and the challenge of engagement (Leach M, Scoones I, Wynne B, Eds), pp. 218-231. London: Zed Books.

Turcanu C et al. 2019. Final report of the ENGAGE project. CONCERT deliverable 9, $94 \mathrm{p}$.

Turcanu C et al. 2020. Stakeholder engagement in radiological protection: Developing theory, practice and guidelines. Radioprotection 55(HS2). https://doi.org/10.1051/radiopro/2020008.

Wehling P. 2012. From invited to uninvited participation (and back?): Rethinking civil society engagement in technology assessment and development. Poiesis Praxis 9(1): 43-60.

Wesselink A, Paavola J, Fritsch O, Renn O. 2011. Rationales for public participation in environmental policy and governance: Practitioners' perspectives. Env. Plan. A: Econ. Space 43(11): 2688-2704.

WHO. 2017. Emergency response framework. Geneva: World Health Organization.

Wynne B. 2007. Public participation in science and technology: Performing and obscuring a political-conceptual category mistake. East Asian Sci. Technol. Soc. 1(1): 99-110.

Young IM. 2001. Activist challenges to deliberative democracy. Pol. Theory 29(5): 670-690.

Zeleznik N et al. 2019a. Rationales and frameworks for stakeholder engagement in radiation protection. CONCERT Deliverable 9.85.

Zeleznik N et al. 2019b. Report on venues, challenges, opportunities and recommendations for stakeholder engagement in emergency and recovery preparedness and response. CONCERT Deliverable D9.90.

Cite this article as: Geysmans R, Zeleznik N, Abelshausen B, Duranova T, Schieber C, Schneider T, Crouail P, Turcanu C, Liutsko L, Cantone MC. 2020. Broadening and strengthening stakeholder engagement in emergency preparedness, response and recovery. Radioprotection 55(HS2): S219-S225 